Chappe Vincent-Arnaud, Keyhani Narguesse, « La fabrique d'un collectif judiciaire. La mobilisation des cheminots marocains contre les discriminations à la SNCF ", Revue française de science politique, 2018/1 (Vol. 68), p. 7-29. Version pré-print

\title{
LA FABRIQUE D’UN COLLECTIF
}

JUDICIAIRE

LA MOBILISATION DES CHEMINOTS MAROCAINS CONTRE LES DISCRIMINATIONS A LA SNCF

\section{Vincent-Arnaud Chappe et Narguesse Keyhani}

Publication en version définitive: Chappe Vincent-Arnaud, Keyhani Narguesse, «La fabrique d'un collectif judiciaire. La mobilisation des cheminots marocains contre les discriminations à la SNCF », Revue française de science politique, 2018/1 (Vol. 68), p. 729. DOI : $\underline{10.3917 / \mathrm{rfsp} .681 .0007}$.

Au cours de la semaine du 23 mars 2015 se tient au conseil de prud'hommes de Paris un procès opposant 848 cheminots à la SNCF. Ces salariés, pour la très grande majorité, marocains ont été recrutés au début des années 1970 directement à proximité de leur lieu d'habitation pour un grand nombre, avec la promesse écrite dans leur contrat de travail d'un traitement à égalité avec leurs futurs collègues français. Comme d'autres employeurs à cette époque, la compagnie ferroviaire recourt à la main-d'œuvre étrangère, en s'appuyant notamment sur une « convention de main-d'œuvre » passée en 1963 entre le France et le Maroc. Ils voient pour la plupart leur carrière s'achever au cours des années 2000, avec une retraite souvent très faible. Ils dénoncent devant la justice du travail une discrimination liée à leur nationalité ou leur origine, avec des conséquences sur leur statut d'embauche - «auxiliaires contractuels » plutôt que «cadres permanents »-, l'évolution de leur carrière, leur pension et un ensemble de droits afférents (santé et transport) ${ }^{1}$. Le jugement en appel rendu le 31 janvier 2018 vient confirmer la condamnation de l'employeur pour discrimination, comme l'avait fait le conseil de prud'hommes deux ans plus tôt. Si ce procès réunit un nombre élevé et unique en France de plaignants en matière de discrimination, il n'est pas en soi une action collective mais l'adjonction d'autant de cas individuels traités plus ou moins simultanément par l'institution judiciaire prud'homale ${ }^{2}$, tout en étant pensé comme une action collective par l'ensemble des

\footnotetext{
${ }^{1}$ Dominique Andolfatto, Marnix Dressen, «Invisibles et dominés. Trois études de cas de "hors statut" dans le secteur ferroviaire : Marocains, maîtres-chiens et pompiers », Communication au $5^{\mathrm{e}}$ Congrès de l'Association française de sociologie, Nantes, 2013.

${ }^{2}$ À la demande notamment de professionnels du droit spécialistes des discriminations, l'action de groupe en la matière a été introduite dans le droit français par la loi du 18 novembre 2016 de «modernisation de la justice du $21^{\mathrm{e}}$ siècle », trop tard donc pour concerner ce contentieux amorcé dès la moitié des années 2000. Cette nouvelle
} 
Chappe Vincent-Arnaud, Keyhani Narguesse, « La fabrique d'un collectif judiciaire. La mobilisation des cheminots marocains contre les discriminations à la SNCF », Revue française de science politique, 2018/1 (Vol. 68), p. 7-29. Version pré-print

demandeurs concernés et par leurs soutiens. En cherchant à saisir simultanément le déroulement de l'action judiciaire et la formation d'un collectif, cet article aborde une question centrale de la science politique: celle des conditions d'existence des collectifs articulés à des revendications dans l'espace public.

Ce cas pose la question des relations entre mobilisations collectives et action judiciaire et de leurs effets sur la formation des collectifs autour d'une cause. La sociologie politique du droit a souligné la limite des portées politiques des actions collectives portées devant les arènes judiciaires ${ }^{3}$. D'autres travaux ont nuancé fortement ces résultats, insistant sur les effets de « libération cognitive » permise par l'action judiciaire ${ }^{4}$; de publicisation des causes ${ }^{5}$; sur la possible mise en série de contentieux au sein d'une cause collective ${ }^{6}$; tout en insistant sur les effets de cadrage plus ou moins restrictifs produits par le recours au droit. L' ${ }^{\prime}$ arme du droit ${ }^{7}$ est maintenant une pièce du répertoire d'action collective bien connue des politistes ${ }^{8}$, aussi bien dans sa capacité à porter une cause politique et à rassembler des acteurs, que dans les contraintes qui l'accompagnent.

L'analyse de l'action judiciaire menée par les cheminots marocains - qualifiés par la presse de «Chibanis » («cheveux blancs » en arabe, en référence à leur âge relativement élevé) -, permet de poursuivre le questionnement porté par ces travaux, en effectuant un double décalage. Il s'agit moins ici de s'intéresser aux conséquences politiques d'une action collective qu'aux effets de cette action sur l'agrégation des individus concernés : que fait le procès - conçu comme « dispositif » de temps long non réductible aux audiences ${ }^{9}$ - à ceux qui s'y engagent, en tant que collectif? Dans quelle mesure la judiciarisation du conflit a-t-elle eu des effets sur la formation et la définition du groupe ? Cette interrogation s'appuie sur les réflexions avancées

modalité procédurale est de plus limitée dans son usage ce qui rend les observateurs sceptiques sur son effectivité et sa généralisation. Certaines actions de groupe ont néanmoins récemment été lancées, notamment en matière de discrimination liée au handicap à la $\mathrm{SNCF}$, mais sans qu'on puisse encore juger de leur réussite. Frédéric Guiomard, «L'action de groupe peut-elle contribuer à lever les freins à l'action contentieuse ? », La revue des droits de l'homme. Revue du Centre de recherches et d'études sur les droits fondamentaux, février 2016.

${ }^{3}$ Gerald N. Rosenberg, The Hollow Hope. Can Courts Bring about Social Change ?, Chicago, University of Chicago Press, 1991 ; Stuart A. Scheingold, The Politics of Rights. Lawyers, Public Policy and Political Change, Michigan, University of Michigan Press, 2004.

${ }^{4}$ Doug McAdam, Political Process and the Development of Black Insurgency, 1930-1970, Chicago, University of Chicago Press, 2010.

${ }^{5}$ Emmanuel Henry, «Le droit comme vecteur de publicisation des problèmes sociaux : effets publics du recours au droit dans le cas de l'amiante », dans Liora Israël, Guillaume Sacriste, Antoine Vauchez, Laurent Willemez, Sur la portée sociale du droit. Usages et légitimité du registre juridique, Paris, CURAPP, PUF, 2005, p. 187-200.

${ }^{6}$ Emmanuelle Fillion, Didier Torny, « De la réparation individuelle à l'élaboration d'une cause collective », Revue française de science politique, 65 (4), août 2015, p. 583-607.

${ }^{7}$ Liora Israël, L'arme du droit, Paris, Presses de Sciences Po, 2009.

${ }^{8}$ Éric Agrikoliansky, «Les usages protestataires du droit », dans Olivier Fillieule, Éric Agrikoliansky, Isabelle Sommier (dir.), Penser les mouvements sociaux. Conflits sociaux et contestations dans les sociétés contemporaines, Paris, La Découverte, 2010, p. 225-243.

${ }^{9}$ Nicolas Dodier, Janine Barbot, «La force des dispositifs », Annales. Histoire, Sciences Sociales, 71 (2), 2016 , p. 421-450. 
Chappe Vincent-Arnaud, Keyhani Narguesse, « La fabrique d'un collectif judiciaire. La mobilisation des cheminots marocains contre les discriminations à la SNCF », Revue française de science politique, 2018/1 (Vol. 68), p. 7-29. Version pré-print

par Michael McCann ${ }^{10}$ : il repère un effet de «catalyse » de la mobilisation portée par l'action judiciaire mais également $\mathrm{d}^{\text {' } ~}$ empowerment »- à un niveau à la fois individuel et collectif. Le processus de «victimisation » par le droit ${ }^{11}$ - entendu comme processus interactionnel de constitution de l'identité de victime - n'irait pas uniquement dans le sens de la « passivité » qui leur est habituellement attribuée ${ }^{12}$ : il aurait la possibilité d'agir sur l'affirmation en tant que collectif de ceux qui s'engagent pour la reconnaissance de leurs droits.

Le deuxième décalage porte sur le type d'espaces sociaux investi dans ce procès. La mobilisation des contractuels marocains concerne le monde du travail, domaine peu investi dans le cas français par la sociologie du droit ${ }^{13}$, à l'exception de travaux portant principalement sur les mobilisations syndicales ${ }^{14}$. Elle porte de plus sur la dénonciation d'une discrimination, catégorie juridique relativement récente renforcée sous l'effet du droit européen depuis 2001, et de plus en plus utilisée comme motif de qualification des injustices contemporaines ${ }^{15}$. Contrairement au cas américain où de nombreuses études portent sur l'évolution de la catégorie juridique de discrimination sous l'effet des différents facteurs ${ }^{16}$ et sur les limites du droit et du tribunal comme ressource pour les victimes ${ }^{17}$, peu de travaux français se sont intéressés à la mobilisation de la catégorie de discrimination au travail ${ }^{18}$. Dans le cas particulier des discriminations ethno-raciales, les recherches insistent sur le caractère dépolitisant du droit ${ }^{19}$,

\footnotetext{
${ }^{10}$ Michael W. McCann, Rights at Work. Pay Equity Reform and the Politics of Legal Mobilization, Chicago, University of Chicago Press, 1994.

${ }^{11}$ Yannick Barthe, Les retombées du passé. Le paradoxe de la victime, Paris, Seuil, 2017.

12 Violaine Roussel, «Les "victimes" : label ou groupe mobilisé ? Éléments de discussion des effets sociaux de la catégorisation », dans Sandrine Lefranc, Lilian Mathieu (dir.), Mobilisations de victimes, Rennes, Presses Universitaires de Rennes, 2015, p. 11-26.

${ }^{13}$ Hélène Michel, « Justice au travail et travail du droit : invitations à une sociologie différenciée du droit et de la justice », Politix, 118, 2017, p. 9-28.

14 Julien Louis, «Qui perd gagne!», Politix, 118, 2017, p. 29-53 ; Jérôme Pélisse, « Judiciarisation ou juridicisation ? Usages et réappropriations du droit dans les conflits du travail », Politix, 86, 2009, p. 73-96; Laurent Willemez, «Quand les syndicats se saisissent du droit: invention et redefinition d'un rôle », Sociétés contemporaines, 52, 2003, p. 17-38.

${ }^{15}$ François Dubet, Olivier Cousin, Éric Macé et al., Pourquoi moi ? L'expérience des discriminations, Paris, Seuil, 2013.

16 Frank Dobbin, Inventing Equal Opportunity, Princeton, Princeton University Press, 2009; Lauren B. Edelman, Working Law. Courts, Corporations and Symbolic Civil Rights, Chicago, University of Chicago Press, 2016.

${ }^{17}$ Ellen Berrey, Robert L. Nelson, Laura Beth Nielsen, Rights on Trial. How Workplace Discrimination Law Perpetuates Inequality, Chicago, University of Chicago Press, 2017.

${ }^{18}$ Cf. néanmoins Vincent-Arnaud Chappe, «Dénoncer en justice les discriminations syndicales : contribution à une sociologie des appuis conventionnels de l'action judiciaire », Sociologie du travail, 55 (3), 2013, p. 302321 ; Évelyne Serverin, Frédéric Guiomard, «Des revendications des salariés en matière de discrimination et d'égalité : les enseignements d'un échantillon d'arrêts extrait de la base JURICA (2007-2010) », Mission de recherche Droit et Justice, 2014.

${ }^{19}$ Didier Fassin, «L'invention française de la discrimination », Revue française de science politique, 52 (4), août 2002, p. 403-423.
} 
Chappe Vincent-Arnaud, Keyhani Narguesse, « La fabrique d'un collectif judiciaire. La mobilisation des cheminots marocains contre les discriminations à la SNCF », Revue française de science politique, 2018/1 (Vol. 68), p. 7-29. Version pré-print

portant une vision individualisante des discriminations plutôt que groupale ou collective ${ }^{20}$, dans le cadre d'un modèle républicain hostile à la reconnaissance de la race comme catégorie d'appréhension des relations de pouvoir et des inégalités ${ }^{21}$. Ces caractéristiques françaises du droit de la non-discrimination, associées à la faible réceptivité des institutions judiciaires françaises aux actions de groupe, semblent être défavorables à la constitution d'un collectif unifié par le procès et l'unicité du tort subi ${ }^{22}$. Il n'en reste pas moins que cette action judiciaire est unique en son genre dans la mesure où elle est la première à rassembler autant de plaignants sur la dénonciation de la discrimination liée à la nationalité et à l'origine. Comment analyser alors la nature de ce collectif ? N'est-il que la réunion limitée dans le temps d'un ensemble de plaignants réunis au sein d'un même dispositif judiciaire, ayant délégué leur pouvoir d'agir à des professionnels et experts parlant en leur nom ? Peut-on repérer des effets spécifiques de cette expérience des discriminations dans l'univers du travail, sur la consolidation du groupe mobilisé ? Une telle expérience peut-elle favoriser une action commune et une mémoire partagée ${ }^{23}$ autour des discriminations ethno-raciales, dans un cadre institutionnel pourtant inhospitalier ${ }^{24}$ ?

L'article s'inscrit à l'intersection de la sociologie des mobilisations collectives et judiciaires et de celle des groupes sociaux. Il s'intéresse à la judiciarisation de la mobilisation pour en cerner les effets sur le devenir du collectif, l'action judiciaire se déployant selon une temporalité particulière, dans une arène de revendications spécifique et mobilisant un répertoire d'action et un langage propres. Il s'attache à expliquer le rôle des acteurs ayant permis d'objectiver les contractuels marocains comme groupe unifié. Si «l'action protestataire constitue un terrain propice au travail identitaire ${ }^{25}, 1$ 'article accorde une attention particulière aux soutiens qui homogénéisent le groupe ${ }^{26}$, notre hypothèse étant que le caractère judiciaire de la trajectoire prise par l'action collective contribue à faire de ce groupe un collectif réel, mais très largement «parlé » par d'autres. L'analyse s'appuie sur une recherche ethnographique menée de façon continue depuis 2015 : elle repose sur l'observation de scènes collectives (procès, réunions associatives, permanences juridiques), sur des entretiens formels et informels avec les plaignants et protagonistes de la mobilisation (syndicalistes, professionnels du droit, militants associatifs) et sur des archives syndicales (tracts, documents de communication

\footnotetext{
${ }^{20}$ Vincent-Arnaud Chappe, « Le cadrage juridique, une ressource politique ? La création de la HALDE comme solution au problème de l'effectivité des normes anti-discrimination (1998-2005) », Politix, 94, 2011, p. 107-130.

${ }^{21}$ Mireille Eberhard, "L’idée républicaine de la discrimination raciste en France », thèse de doctorat, Université Paris-Diderot, 2006.

${ }^{22}$ La part des contentieux en appel engageant des « collections d'actions individuelles » sont ainsi relativement rares dans le contentieux en matière de discrimination, même si elles peuvent avoir une influence importante. Cf. É. Serverin, F. Guiomard, «Des revendications des salariés... », op. cit., p. 171.

${ }^{23}$ Sandrine Lefranc, Lilian Mathieu, « De si probables mobilisations de victimes », dans S. Lefranc, L. Mathieu (dir.), Mobilisations de victimes, op. cit., p. 11-26.

${ }^{24}$ Joan Stavo-Debauge, «Tu ne catégoriseras point ! , Carnets de bord, 6, 2003.

${ }^{25}$ Érik Neveu, Sociologie des mouvements sociaux, Paris, La Découverte, 2015, p. 78.

${ }^{26}$ Rogers Brubaker, «Au-delà de 1'“identité" », Actes de la recherche en sciences sociales, 4, 2001, p. 66-85 ; Martina Avanza, Gilles Laferté, «Dépasser la "construction des identités”? Identification, image sociale, appartenance », Genèses, 61, 2005, p. 134-152.
} 
Chappe Vincent-Arnaud, Keyhani Narguesse, « La fabrique d'un collectif judiciaire. La mobilisation des cheminots marocains contre les discriminations à la SNCF », Revue française de science politique, 2018/1 (Vol. 68), p. 7-29. Version pré-print

interne, etc.) concernant les prémisses de cette mobilisation.

La première partie restitue la dynamique de judiciarisation comme moment de réappropriation par les professionnels du droit d'une mobilisation initialement portée par un petit groupe de cheminots marocains et les syndicats. Elle retrace l'histoire de la mobilisation, les modes d'organisation du collectif, de socialisation qui y sont liés, et le rôle joué par des intermédiaires dans le maintien d'un groupe unifié autour de la procédure judiciaire. La deuxième partie s'intéresse à la traduction du vécu discriminatoire dans la grammaire de l'arène judiciaire. Elle montre comment le procès cadre le déroulement de la mobilisation, mais également le type de narration produit sur l'injustice vécue par les plaignants. Sont mis ainsi en avant les tensions descriptibles au cours des audiences entre une vision individualisée et une vision collective des discriminations, renvoyant à une opposition entre vision formelle et vision matérielle du droit de la non-discrimination. La troisième partie porte sur les prises de paroles des plaignants dans le processus judiciaire et montre comment elles favorisent la subjectivation d'acteurs restés jusque-là en retrait. Elle revient sur les limites de ce mouvement, contrariés par des tendances à la dépossession et à la dépolitisation de l'affaire poussées par les professionnels du droit en charge de la stratégie judiciaire.

\section{Les conditions initiales de la formation d'un groupe de plaignants}

L'existence d'un groupe objectif en termes de caractéristiques socio-démographiques et statutaires ne peut suffire à expliquer en elle-même l'émergence d'une mobilisation ${ }^{27}$. Les cheminots marocains recrutés au début des années 1970 forment bien un groupe spécifique au sein du personnel ; pourtant, il faut attendre le début des années 2000 pour qu'une association francilienne investisse cette question, avant qu'une action judiciaire ne soit entamée en 2004.

Le passage d'un répertoire d'action à l'autre donne à voir diverses logiques de définition du groupe. La judiciarisation de la mobilisation montre comment un groupe géographiquement dispersé est devenu un groupe dénombrable de plaignants. La mobilisation de plaignants repose sur un travail d'identification des porteurs potentiels de griefs et de définition de la nature des injustices subies ${ }^{28}$, par des acteurs parfois extérieurs ${ }^{29}$. La judiciarisation de la mobilisation invite par ailleurs à être attentifs à la dimension séquentielle de ce travail ${ }^{30}$ et aux ressources expliquant la capacité à recourir au droit. Trois facteurs ont renforcé l'action judiciaire et la formation d'un groupe unifié de plaignants : la mobilisation de ressources syndicales (A), la socialisation communautaire (B) et enfin les conditions de l'organisation même de l'action sur le long terme $(\mathrm{C})$.

\footnotetext{
${ }^{27}$ Johanna Siméant, La cause des sans-papiers, Paris, Presses de Sciences Po, 1998.

${ }^{28}$ S. Lefranc, L. Mathieu, Mobilisations de victimes, op. cit.

${ }^{29}$ M. Avanza, G. Laferté, «Dépasser la “construction des identités” ?.. », art. cité. ; R. Brubaker, « Au-delà de 1'“identité" ", art. cité.

${ }^{30}$ William L. F. Felstiner, Richard L. Abel, Austin Sarat, « The Emergence and Transformation of Disputes : Naming, Blaming, Claiming », Law \& Society Review, 15 (3-4), 1980, p. 631-654.
} 
Chappe Vincent-Arnaud, Keyhani Narguesse, « La fabrique d'un collectif judiciaire. La mobilisation des cheminots marocains contre les discriminations à la SNCF ", Revue française de science politique, 2018/1 (Vol. 68), p. 7-29. Version pré-print

\section{L'action syndicale et la cause des contractuels de la SNCF}

Contrairement aux « groupes circonstanciels » nés à la faveur d'accidents collectifs ${ }^{31}$, les cheminots marocains peuvent s'appuyer sur une même appartenance catégorielle: l'inscription originelle au statut PS25. La commune appartenance à ce statut n'a pourtant pas facilité en soi la naissance d'une action collective. Les premières remises en question du statut semblent remonter aux années 1990 et sont circonscrites au niveau local, parfois à l'échelle d'une gare. Des contractuels interrogent leur supérieur hiérarchique, notamment leurs chefs de gare, pour se plaindre d'un refus de formation, d'un rythme d'avancement qui parait injuste, du fait de ne jamais voir leur nom inscrit au tableau des notations. D'autres racontent avoir écrit à leur député. Si la plupart des cheminots rencontrés relatent s'être plaint de façon plus ou moins formelle auprès de leur supérieur hiérarchique, il faut attendre le tournant des années 2000 pour que ces dénonciations individuelles et strictement locales trouvent un appui organisationnel.

L'association Ismaïlia, première association à dénoncer les inégalités de traitement entre les contractuels marocains et leurs collègues français du cadre permanent est créé en 1999 à Bondy autour d'un petit nombre de cheminots du Bourget dont notamment Ahmed Katim - son président -, de la Gare du Nord et de Gare de l'Est. Elle offre aussi un lieu de sociabilité aux cheminots marocains de la région parisienne, des cours de langue et d'aide aux devoirs pour leurs enfants. Ses fondateurs expliquent pourtant la création de l'association par la prise de conscience à l'occasion des premiers départs à la retraite, du montant dérisoire de leurs pensions à venir. À travers l'évocation rituelle de cette « découverte », ils écrivent l'histoire d'une prise de conscience collective des effets concrets de leur statut, tout en l'inscrivant dans une histoire plus longue : celle de l'expérience silencieuse des discriminations, devenues intolérables.

La plupart des fondateurs de l'association sont syndiqués (à FO, à la CGT et à SUD Rail) et certains ont eu des responsabilités syndicales, ce qui facilite l'alerte d'autres syndicalistes. C'est en mobilisant des modes d'action classiques que Sud Rail conteste le statut PS25: information, lancement d'une pétition nationale, mobilisation des différentes centrales syndicales, manifestations mensuelles à Paris. Bien qu'elle dénonce la " préférence nationale », c'est à une problématisation plus générale de la situation des contractuels de la SNCF que s'attelle le syndicat. La dynamique de la protestation a ainsi été facilitée par ces appuis disponibles dans cet univers professionnel et dont l'accès est favorisé par la proximité professionnelle et l'expérience syndicale des membres de l'association. Contrairement à d'autres univers où les cadres cognitifs dominants peuvent constituer des freins à l'imputation de la cause et à l'identification d'un responsable ${ }^{32}$, l'unicité de l'employeur, du statut originel et le caractère routinisé du répertoire syndical sont favorables à la dynamique protestataire.

Ces actions aboutissent le 5 janvier 2004 à l'occupation spectaculaire du siège de la $\mathrm{SNCF}$, à laquelle participent des cadres de Sud-Rail et près de 150 cheminots marocains. En

\footnotetext{
${ }^{31}$ Jean-Paul Vilain, Cyril Lemieux, «La mobilisation des victimes d'accidents collectifs : vers la notion de “groupe circonstanciel” », Politix, 44, 1998, p. 135-160.

32 À propos des freins à la transformation des «risques du travail » en préjudice, dans le cas de l'exposition aux pesticides des agriculteurs, cf. Jean-Noël Jouzel, Giovanni Prete, « De l'intoxication à l'indignation : le long parcours d'une victime des pesticides », Terrains \& travaux, 22, 2013, p. 59-76.
} 
Chappe Vincent-Arnaud, Keyhani Narguesse, « La fabrique d'un collectif judiciaire. La mobilisation des cheminots marocains contre les discriminations à la SNCF », Revue française de science politique, 2018/1 (Vol. 68), p. 7-29. Version pré-print

avril, s'ouvrent des négociations pour la réécriture du statut à laquelle participent cadres syndicaux et cheminots syndiqués d'Ismaïlia. L'obtention en septembre 2004 d'un droit à la préretraite, d'une quatrième classe, d'une carte de circulation et de droits médicaux plus importants sont perçus comme un succès pour les soutiens syndicaux qui ont tout au long de la mobilisation dénoncé à la fois le sort des cheminots étrangers et remis en cause le statut de contractuels. Parallèlement, l'association engage vers 2003 une action judiciaire. Avec le soutien continu d'un cadre permanent et syndicaliste de Sud-Rail, Bob, le noyau de cheminots franciliens s'engage dans une campagne d'identification et de comptabilisation des PS25 dans toute la France. Initié par voie syndicale dès le début des années 2000, ce travail se poursuit avec le déplacement physique du président de l'association Ahmed-Katim et de son soutien de Sud-Rail. Au fil de leur tour des grandes gares de France, ils élaborent un listing qui permet de repérer les cheminots concernés.

La socialisation communautaire : des plaignants marocains

À Malakoff, Creil, Villeneuve-la-Garenne, Metz, Strasbourg, Mulhouse ou encore à Caen et au Havre, existent de longue date des formes d'interconnaissance et de sociabilité communautaire locales et préalables à la mobilisation sur lesquelles ce travail d'identification des PS25 peut s'appuyer. Elles trouvent leur origine dans les conditions mêmes d'entrée dans l'entreprise. Souvent recrutés directement au Maroc et collectivement, ils sont nombreux à l'avoir été dans la même région de leur pays d'origine. Acheminés par petits groupes depuis le Maroc, ils ont ensuite été orientés dans les différentes gares françaises et logés quelques années dans des foyers de travailleurs. De nombreuses familles de contractuels marocains s'installent ensuite dans des logements HLM de la SNCF. Dans les entretiens, des noms de «cités SNCF » reviennent souvent: La Sablière, la cité SNCF de Malakoff, etc. En raison même de leur inscription au statut PS25 qui implique l'occupation de postes entre les trois échelons les plus bas de la hiérarchie des emplois cheminots, ils travaillent souvent ensemble, aux postes les plus pénibles (sur les voies, en gare de triage, etc.). C'est donc le cadre de travail, souvent celui du logement, et en amont, les conditions d'immigration qui expliquent que leur socialisation professionnelle et en dehors du travail, soit en grande partie communautaire. Enfin, à l'âge de la retraite, cette sociabilité se poursuit par les allers-retours entre la France et le Maroc où ils séjournent une partie de l'année.

Cette forme de socialisation a constitué une ressource inattendue : en 2009, alors que les membres de l'association font face à l'échec d'une première action judicaire, l'un d'entre eux, B. rencontre Abdelkader Bendali au Maroc. C'est en échangeant autour de la mobilisation avec des amis dans sa région d'origine à Oujda, où il séjourne régulièrement depuis sa retraite, que B. est présenté à A. Bendali qui très vite lui propose son expertise. Cet homme d'une cinquantaine d'années se présente en effet comme un universitaire formé en partie en France en économie et en droit, ayant enseigné dans les deux pays et alors en poste à Oujda. De retour en région parisienne, B. présente le « professeur » aux autres membres du bureau. Il accepte de venir en soutien de l'affaire au nom de l'importance de la cause mais aussi de son intérêt juridique. 
Chappe Vincent-Arnaud, Keyhani Narguesse, « La fabrique d'un collectif judiciaire. La mobilisation des cheminots marocains contre les discriminations à la SNCF », Revue française de science politique, 2018/1 (Vol. 68), p. 7-29. Version pré-print

Au sein de la mobilisation jusque-là organisée par les membres d'Ismaïlia et des membres de Sud-Rail, son profil est atypique : inconnu des cheminots marocains avant 2009, il l'est également des syndicats. S'il est éloigné de la culture syndicale, il revendique une connaissance du monde judiciaire et des arcanes du droit français sans être à proprement parler un professionnel du droit. C'est pourtant la fonction qu'il parvient à incarner en s'inscrivant à l'interface de l'association, des plaignants et du cabinet d'avocats. La distance sociale qui le sépare des membres de l'association est renforcée par l'ethos professoral qu'il entretient volontiers et avec succès, toujours habillé en costume et portant une cravate, maniant habilement la langue en alternant entre l'arabe et le français, et rappelant régulièrement ses fonctions universitaires au Maroc. Interlocuteur privilégié des avocats dont il rapporte les directives à l'association, il en est devenu le gatekeeper accepté par les plaignants, probablement en raison des effets de légitimation que suscite sa présence sur les cheminots compatriotes.

La socialisation communautaire constitue également un facteur d'accroissement du groupe des plaignants sur le long terme, notamment à partir des années 2010 : en 2005, l'association avait rassemblé 60 dossiers, 360 en 2010, 745 en 2012, 832 en 2015. La victoire prud'homale ouvre « un cycle de production de nouveaux recours ${ }^{33}$ : en France, comme au Maroc, les plaignants communiquent ce succès dans leur entourage local et familial, favorisant « un enrôlement par la proximité » ${ }^{34}$ et aidés en cela par les médias français et marocains mais aussi ceux de l'immigration marocaine en France. Au printemps 2017, alors que les premiers groupes de plaignants passaient en appel, on comptait près de 300 nouveaux plaignants ayant introduit un recours aux prud'hommes.

Le travail bureaucratique d'une association : des plaignants au guichet de la mobilisation

Les acteurs peu familiers de l'action judiciaire (faible capital social pour les uns, spécialisation dans d'autres formes de mobilisations pour les autres) qui ont joué un rôle moteur dans son amorce, l'entretiennent également sur le long terme à travers l'organisation de permanences hebdomadaires et de réunions publiques. Animées par les membres du bureau avec la participation régulière de A. Bendali, elles se tiennent dans les locaux de Sud-Rail, devenu pourvoyeur de ressources. La régularité de ces permanences et les scènes qui s'y jouent permettent d'assurer la mobilisation sur le long terme et de préparer les audiences à venir tout en unifiant un groupe croissant de plaignants. Elles ont en ce sens un rôle important dans « la fabrique » des plaignants. Par les interactions qui s'y jouent, ces permanences sont aussi le lieu où sont définis le rôle et la place des plaignants dans la mobilisation.

Les permanences se tiennent le lundi de 9 heures à 12 heures, dans les locaux de SudRail, près de la Gare de l'Est, dans une salle organisée pour accueillir des réunions syndicales,

\footnotetext{
${ }^{33}$ Emmanuelle Fillion, Didier Torny, « De la réparation individuelle à l'élaboration d'une cause collective : l'engagement judiciaire des victimes du distilbène », Revue française de science politique, 65 (4), août 2015, p. 583-607, dont p. 596.

${ }^{34}$ Annie Collovald, Lilian Mathieu, « Mobilisations improbables et apprentissage d'un répertoire syndical », Politix, 86, 2009, p. 119-143.
} 
Chappe Vincent-Arnaud, Keyhani Narguesse, « La fabrique d'un collectif judiciaire. La mobilisation des cheminots marocains contre les discriminations à la SNCF », Revue française de science politique, 2018/1 (Vol. 68), p. 7-29. Version pré-print

mais qui trouve ici un autre usage : les membres du bureau et A. Bendali s'installent derrière l'une des tables qui apparaît alors comme un guichet les séparant des plaignants. Ce sont ces permanences qui organisent à distance la relation entre conseils et plaignants en prenant en charge le volet administratif du travail de l'avocate Clélie de Lesquen. La tenue de ces permanences entretient une distance physique, sociale et symbolique avec le professionnel du droit et alimente la dimension groupale de l'action judiciaire qui formellement reste composée de centaines de plaintes individuelles.

Les membres du bureau accueillent les cheminots qui viennent de toute la France : rarement seuls, ils rejoignent le local depuis les différentes gares parisiennes, souvent par petits groupes. Une demi-heure à peine après l'ouverture de la permanence, la salle est souvent bondée. Certaines permanences accueillent plus d'une trentaine de personnes. Les premiers arrivés peuvent s'asseoir, les autres attendent debout. Une queue se forme pour accéder à la table où les membres du bureau et $\mathrm{A}$. Bendali les accueillent et vérifient les dossiers. Chaque plaignant potentiel apporte contrat de travail, fiches de paie, relevés de carrières, notification de départ à la retraite, attestation de formation, etc. Tous ces documents sont recueillis, ordonnés, et rangés dans un dossier qui constitue l'ensemble des preuves sur lesquels l'avocate s'appuie pour construire l'affaire. La tenue régulière de ces permanences permet l'accroissement du groupe des plaignants et la consolidation continue des plaintes. En devant passer par la permanence, les cheminots ne peuvent devenir plaignant qu'en rejoignant le groupe. Bien que reçus individuellement, les plaignants sont de temps à autre abordés collectivement par A. Bendali, parfois en français, souvent en arabe. Il explique les raisons pour lesquelles les dossiers doivent être constitués attentivement, ce que l'avocate pourra établir grâce à telle pièce, précise que les plaignants doivent avoir reçu telle notification de l'administration judiciaire. Il explique aussi les décisions des juges et informe plus généralement sur le cours de l'action judiciaire. C'est donc à la fois un lieu où il informe les plaignants de l'action en cours et où les dossiers sont construits, complétés et standardisés.

L'ambiance des permanences oscille entre accueil bureaucratique des plaignants et familiarité : certains plaignants arrivent tôt et s'installent dans la salle de réunion durant toute la permanence, alors que leur dossier est déjà complet. Ils accompagnent un ami, restent et discutent avec d'autres plaignants. Les permanences offrent un espace de collectivisation ponctuelle de l'expérience judiciaire ${ }^{35}$. Dans le même temps, elles consolident la stricte division des tâches entre l'ensemble des plaignants d'une part, l'association et les professionnels du droit d'autre part. Jusqu'aux audiences de 2015, une grande majorité de plaignants n'ont pas de contact avec l'avocate. Ce rôle d'interface incarné par Ismaïlia et A. Bendali s'explique pour eux par la nécessité de rationaliser le recueil et la gestion des plaintes individuelles. Mais en soulignant régulièrement ce rôle, A. Bendali rappelle également à l'ordre les plaignants sur leur place et l'obéissance qu'ils doivent témoigner, voire n'hésite pas à élever la voix contre eux en s'appuyant sur son aura professorale. La frontière entre

\footnotetext{
${ }^{35}$ E. Fillion, D. Torny, « De la réparation individuelle à l'élaboration d'une cause collective », art. cité.
} 
Chappe Vincent-Arnaud, Keyhani Narguesse, « La fabrique d'un collectif judiciaire. La mobilisation des cheminots marocains contre les discriminations à la SNCF », Revue française de science politique, 2018/1 (Vol. 68), p. 7-29. Version pré-print

profanes et professionnels du droit ${ }^{36}$ prend ici une forme originale. A. Bendali est perçu comme un professionnel du droit et comme stratège de l'action judiciaire, mais également comme marocain, ce qui semble clairement favoriser l'identification de nombreux plaignants qui y trouvent un faire-valoir. Le processus de judiciarisation de la mobilisation crée ainsi manifestement un groupe de plaignants tenus à distance voire dans la passivité. Dans le même temps, l'identification communautaire et nationale à cet acteur atypique semble permettre à ces acteurs de gagner en dignité et ce malgré la violence symbolique exercée sur eux.

\section{Dénoncer les discriminations collectives dans l'arène judiciaire}

La transformation de la mobilisation collective en mobilisation judiciaire a des conséquences en termes de cadrage de l'action, concernant notamment son format, les compétences qu'elle nécessite, ses contraintes, la narration du tort sur lequel elle s'appuie et la redistribution des responsabilités qu'elle provoque ${ }^{37}$. Le procès implique l'entrée dans un dispositif spécifique, muni d'une temporalité propre ${ }^{38}$, qui contraint fortement le déroulement des événements (A). Le procès institue également une grammaire propre à l'arène judiciaire. Il implique une traduction dans le langage formel du droit qui n'est pas sans incidence sur la production d'une représentation spécifique de ce qu'est la discrimination. L'opération juridique de qualification du fait discriminatoire se fait alors en tension entre des interprétations différentes: une interprétation individualiste de la discrimination par opposition à une interprétation plus collective (B), qui renvoie à une opposition entre approche formelle et matérielle du droit $(\mathrm{C})$.

\section{Une mobilisation structurée par la temporalité judiciaire}

La décision de saisir la justice implique un alignement de la mobilisation au calendrier dicté de façon hétéronome par l'institution judiciaire. Le procès est en effet fortement structuré par une trame temporelle articulée autour de séquences étalées dans le temps définissant le fonctionnement normal de la procédure. Le déroulé de ces séquences dépend à la fois de la voie judiciaire choisie, des choix effectués par les acteurs et des décisions rendues par l'institution judiciaire.

En 2004, sur les conseils d'un premier avocat, l'association s'engage devant le Conseil d'État pour contester la légalité du statut PS25 sur lequel s'appuie la gestion des cheminots marocains contractuels. Plutôt que l'entreprise, c'est donc l'État qui est visé par cette procédure en tant qu'il est le producteur des décrets sur lesquels se fonde la régulation différentielle du personnel. Le Conseil d'État rejette le recours judiciaire, confirmant la légalité formelle du statut des auxiliaires.

\footnotetext{
36 Pierre Bourdieu, «La force du droit [Éléments pour une sociologie du champ juridique] », Actes de la recherche en sciences sociales, 64, 1986, p. 3-19.

${ }^{37}$ Jean-Noël Jouzel, Giovanni Prete, « Devenir victime des pesticides : le recours au droit et ses effets sur la mobilisation des agriculteurs Phyto-victimes », Sociologie du travail, 56 (4), 2014, p. 435-453.

${ }^{38}$ N. Dodier, J. Barbot, « La force des dispositifs », art. cité.
} 
Chappe Vincent-Arnaud, Keyhani Narguesse, « La fabrique d'un collectif judiciaire. La mobilisation des cheminots marocains contre les discriminations à la SNCF », Revue française de science politique, 2018/1 (Vol. 68), p. 7-29. Version pré-print

Ce premier échec marque un coup d'arrêt dans la mobilisation judiciaire. Celle-ci est interrompue jusqu'à 2010 avant d'être reprise en main à la suite de la rencontre de l'association avec A. Bendali. En collaboration avec un avocat, il décide de réorienter l'action vers la justice prud'homale, avec l'argument que le contentieux ne concerne pas la légalité du statut en tant que tel mais la légalité de l'exécution du contrat de travail. Le déroulé de la procédure prud'homale est articulé autour de multiples étapes : bureau de conciliation à la recherche d'une solution amiable entre les parties ; puis bureau de jugement devant quatre conseillers élus - des syndicats de salariés et patronaux. En cas d'égalité des voix devant cette formation, une nouvelle audience se tient devant un juge départiteur, cette fois-ci professionnel, qui tranche et émet un jugement définitif de première instance. Selon le résultat, les parties peuvent faire appel de la décision, qui est alors renvoyée devant la Chambre sociale de la Cour d'appel. La décision est ensuite susceptible d'être portée en Cassation avant d'être éventuellement renvoyée devant la Cour d'appel, voire exceptionnellement devant des chambres supranationales.

Dans le cas de cette mobilisation, cette dilution temporelle est amplifiée par la nécessité pour le tribunal de gérer l'ampleur et le caractère collectif du dossier. Celui-ci ne se présente pas d'un bloc, mais comme la succession d'un ensemble de seize groupes engagés successivement - à mesure que le collectif grossit - dans la procédure judiciaire. L'étape de la conciliation s'étale entre 2007 et 2012 et échoue systématiquement pour tous les groupes. Les différents groupes passent ensuite successivement devant le bureau des jugements, certaines audiences étant repoussées de plusieurs mois en raison de demandes acceptées de report formulées par la SNCF. L'ensemble des groupes sont regroupés au cours d'une semaine marathon d'audiences devant le juge départiteur qui se tient en mars 2015. La délibération des audiences est rendue le 21 septembre 2015. Elle aboutit à une condamnation pour la discrimination de la majorité des 848 cheminots plaignants, condamnation pour laquelle la SNCF fait appel. Les dates du procès d'appel sont fixées aux 15 et 16 mai 2017 : cette fois-ci, les audiences sont réunies sur deux jours divisés quatre sessions. La condamnation de l'entreprise est confirmée le 31 janvier 2018, plus de quatorze ans après le début de l'affaire judiciaire ${ }^{39}$. Les plaignants ne comprennent pas les raisons de ces délais, comme l'exprime par exemple leur ressentiment après la décision de la SNCF de faire appel :

«En arrivant au local à 10 heures, je commence à discuter avec un membre de la "garde rapprochée" de l'association de la décision de l'appel, qu'il a appris hier soir, par la presse. Il se dit surpris et déçu de cette décision, car selon lui en général la SNCF accepte les décisions de justice, et de plus le premier jugement était "équitable". Il me dit avoir reçu un appel désemparé la veille soir à minuit d'un Marocain : l'homme en question "tremblait" au téléphone, il a dû le rassurer. » (Journal de terrain du 24 novembre 2015)

La décision de la SNCF vient également heurter l'espérance de vie réduite de beaucoup de cheminots, en mauvaise santé et pour beaucoup moins âgés qu'ils ne le paraissent. L'appel des noms au début des audiences fait ressortir le taux élevé de décès précoces des cheminots marocains :

"L'appel des noms en début d'audience commence à 8 h 57. À l'appel d'un nom, quelqu'un répond dans la salle "il est au Maroc", ce qui provoque les rires du public. Un autre nom sera accueilli par un "il est mort", un autre

${ }^{39}$ En 2016, la durée moyenne des affaires terminées au conseil de prud'hommes est de 14,7 mois, toute affaire confondue. Cf. Ministère de la Justice, Les chiffres clés de la Justice 2017, 2017, p. 13. 
Chappe Vincent-Arnaud, Keyhani Narguesse, « La fabrique d'un collectif judiciaire. La mobilisation des cheminots marocains contre les discriminations à la SNCF ", Revue française de science politique, 2018/1 (Vol. 68), p. 7-29. Version pré-print

encore par "il est parti", ou malade, ou hospitalisé... [...] se donnent ainsi à voir les ravages du temps sur une population fragile et usée par le travail. » (Journal de terrain du 24 mars 2015)

\section{Le procès : une approche collective contrariée par la forme du procès prud'homal}

Dans l'organisation même du procès aux prud'hommes, on observe les effets de cette pratique de division du collectif en seize groupes, à travers la succession au cours des années 2013 et 2014 d'une dizaine d'audiences devant le bureau de jugements, avec à chaque fois la même décision in fine de renvoi devant le juge départiteur. Le formalisme de la procédure judiciaire ne reconnaît ainsi qu'à moitié le caractère collectif de la procédure dans la mesure où il y a bien des regroupements effectués par cinquantaine de plaignants, mais sans qu'ils soient traités sous la forme d'un collectif unique. Après que tous les groupes aient été renvoyés en départage, la tenue du procès devant le juge départiteur montre à nouveau l'inadéquation institutionnelle de la justice au déploiement d'une telle mobilisation collective. Le respect de la procédure formelle empêche le regroupement en une audience unique des seize groupes. Les règles judiciaires obligent à reprendre la division instaurée en amont dans la procédure prud'homale, avec comme résultat un étalement du procès plaidé treize fois en une semaine, cette procédure obligeant à rejouer treize fois la même scène. L'inadéquation de l'institution judiciaire à la tenue d'un procès collectif se donne à voir jusque dans son organisation matérielle. Le conseil de prud'hommes doit recourir à la plus grande salle qui accueille normalement les référés, et permet de faire tenir à la première audience, près de 200 personnes dont la moitié debout, pour une salle théoriquement de 80 places.

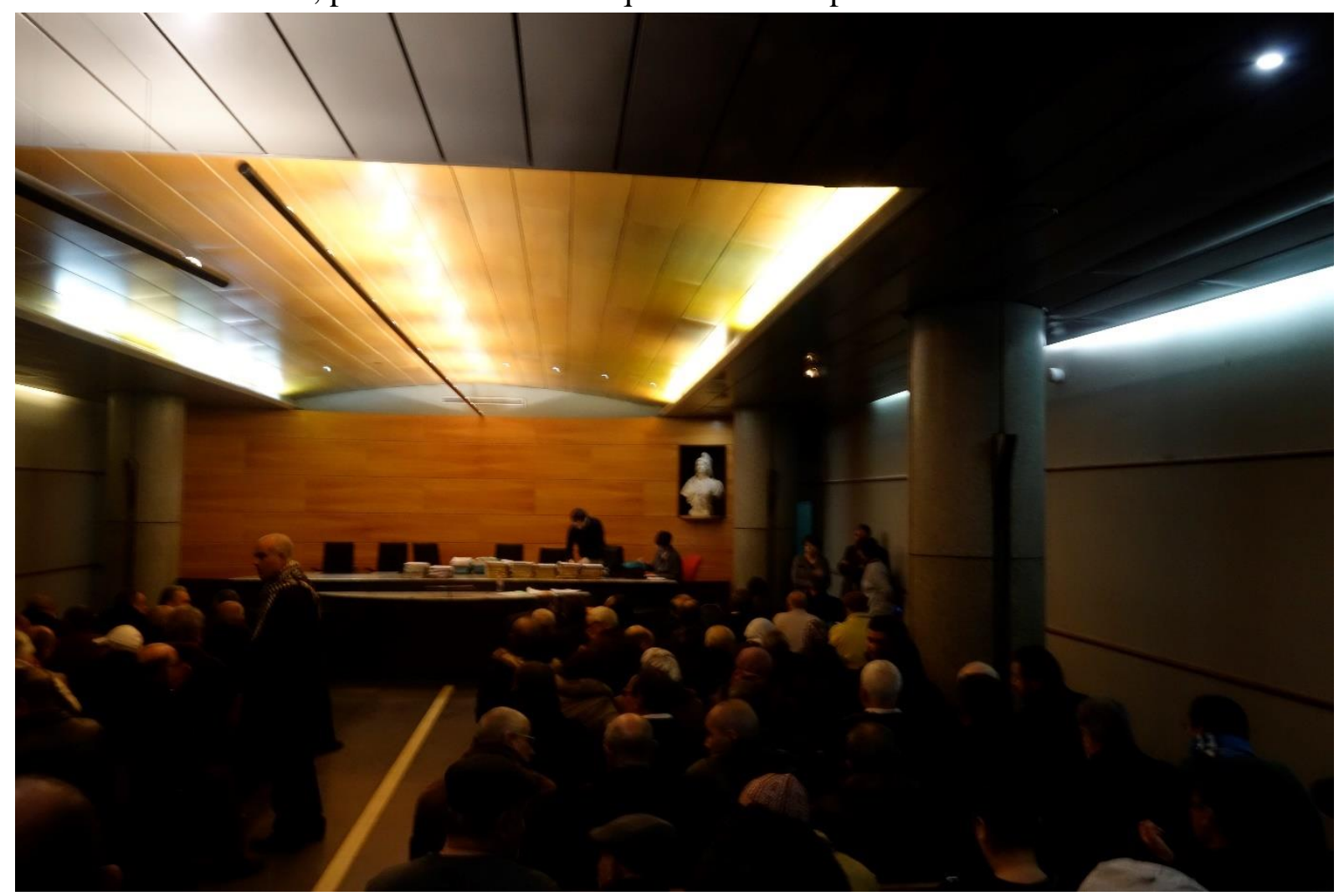

La tenue du procès en appel montre une anticipation plus grande des contraintes propres 
Chappe Vincent-Arnaud, Keyhani Narguesse, « La fabrique d'un collectif judiciaire. La mobilisation des cheminots marocains contre les discriminations à la SNCF », Revue française de science politique, 2018/1 (Vol. 68), p. 7-29. Version pré-print

à ce gigantesque procès. Les seize groupes sont réorganisés en quatre, réunis cette fois-ci dans l'objectif d'atteindre une certaine homogénéité des parcours, contrairement à la stratégie de première instance. Les plaidoiries des avocats, réparties en quatre audiences sur deux jours, ne se répètent plus d'une audience à l'autre, mais portent à chaque fois sur des points différents. La salle est également plus grande, ce qui ne l'empêche d'être à nouveau bondée.

Une autre problématique liée à cette dimension collective est l'existence d'un hiatus entre l'allégation d'une matrice commune du processus discriminatoire à tous les plaignants, et l'existence objective d'une pluralité de catégories de plaignants. Si la très grande majorité des plaignants sont marocains, une très faible minorité venant d'autres pays du Maghreb ou d'Afrique subsaharienne. De plus, la moitié à peu près ont acquis au cours de leur carrière la nationalité française et, selon l'âge de cette acquisition, certains ont pu rejoindre tardivement le « cadre permanent ». Les cheminots se distinguent également par leur avancée professionnelle, plus ou moins existante, par leurs différentes localisations, par l'acceptation ou non d'un dispositif de préretraite après 2004. Enfin, une petite minorité de cheminots l'a été sous un statut spécial de «détachés » de l' «Office national des chemins de fer » marocain. Cette diffraction des cas n'est pas sans effet sur l'unicité du collectif : les différences de statut et de situations ont des conséquences sur la carrière et les droits des concernés.

Pour pallier ce problème, $\mathrm{A}$. Bendali affirme avoir fait le choix de mélanger en première instance au sein des groupes constitués les différents cas, afin de montrer que, malgré la pluralité des situations, une discrimination commune existe, liée à l'origine nationale et en partie indépendante du statut. L'argument est par exemple mobilisé que la discrimination perdure même dans les cas où les cheminots ont acquis la nationalité française et le statut de cadre permanent. Cette manière de faire apparaît comme un moyen de montrer le caractère commun et collectif de la discrimination en dépit des différences objectives. La plaidoirie de C. de Lesquen, l'avocate des cheminots, est de fait de portée collective : elle retrace l'histoire dans laquelle s'inscrit la discrimination, les contextes d'immigration, les différentes manifestations des inégalités de traitement. Elle mobilise de façon ponctuelle des références sociologiques, citant par exemple Maryse Tripier à qui elle attribue le «mythe du retour » comme horizon des destins d'immigration. Parallèlement, elle exploite un argument de registre statistique, habituel dans les procès de discrimination ${ }^{40}$, en détaillant les données du bilan social pour montrer le caractère anormal, à un niveau collectif, de la stagnation de carrière des cheminots marocains.

Cette faible individualisation constitue l'angle principal d'attaque de l'avocat de la SNCF. Il dénonce le caractère collectif de la plaidoirie, affirme que les arguments utilisés sont de nature générale en renvoyant à des grands principes, et affirme qu'il faudrait faire 848 démonstrations spécifiques du fait discriminatoire :

Le principal avocat de la SNCF affirme qu'“aujourd'hui comme hier on plaide de façon générale et pas sur les dossiers individuels. S'il y avait des dossiers individuels, j’aurais pu préparer ma défense”, ce qui aurait nécessité alors de "sortir les pièces des mètres cubes de dossiers". À une autre audience la même journée, le deuxième avocat de la SNCF précise également qu'on ne se situe pas dans le cadre d'une action de groupe, "très à la mode”, mais

\footnotetext{
${ }^{40}$ Olivier Leclerc, «Égalité des personnes et modes de preuve : à propos des usages du raisonnement statistique dans la preuve des discriminations », dans Georges Borenfreund, Isabelle Vacarie (dir.), Le droit social, l'égalité et les discriminations, Paris, Dalloz, 2013, p. 77-94.
} 
Chappe Vincent-Arnaud, Keyhani Narguesse, « La fabrique d'un collectif judiciaire. La mobilisation des cheminots marocains contre les discriminations à la SNCF », Revue française de science politique, 2018/1 (Vol. 68), p. 7-29. Version pré-print

dans des actions individuelles. Il faudrait alors regarder les cas à un niveau individuel - toujours le même argument de l'individualisation - en respectant le contradictoire. (Journal de terrain du 24 mars 2015)

En poussant à la singularisation des plaintes ${ }^{41}$, il s'appuie donc sur le caractère individualiste de la grammaire juridique pour dénoncer la faute que serait la plaidoirie adverse. Du côté des cheminots, le contre-argument est que cette individualisation existe bien dans les dossiers où chaque cas a été exploré en profondeur, l'oralité ne servant qu'à montrer la systématicité et la répétition des discriminations :

«Dans sa plaidoirie, l'avocate des cheminots dit qu'elle en a assez que le dossier soit traité de vulgaire "class action". Elle trouve cela méprisant par rapport au travail de K. qui a rassemblé les informations, de celui du professeur A. Bendali et de celui des avocats. Selon elle, toutes les données sont individualisées, et pour chaque plaignant il y a un tableau récapitulatif qui montre la discrimination. » (Journal de terrain du 26 mars 2015)

L'argument de la SNCF - et sa validation a contrario du côté des plaignants - montre ainsi la difficulté à sortir d'une approche au cas par cas dans le cas d'un procès collectif devant la justice du travail. L'avocate tente d'y parvenir à travers un travail de montée en généralité par mise en série des cas ${ }^{42}$; ce travail est toujours néanmoins susceptible de laisser prise à la critique dans la mesure où le parcours inverse de ré-individualisation, c'est-à-dire de réindexation de la dénonciation au niveau individuel, ne serait pas possible.

\section{Une conception de la non-discrimination entre droit formel et droit matériel}

La tension mise en avant entre approche individuelle et collective renvoie plus largement à la distinction wébérienne classique entre droit formel et matériel ${ }^{43}$, telle qu'elle a été retravaillée par Nicholas Pedriana et Robin Stryker dans le cadre du droit de la nondiscrimination ${ }^{44}$. Les deux chercheur.e.s opposent une approche formelle de ce droit, qui protège des comportements explicitement discriminatoires (c'est-à-dire s'appuyant sur un critère prohibé par la loi, comme par exemple l'origine ou le sexe) à une approche matérielle ou substantielle, où l'interprétation du droit vise à poursuivre les objectifs finals de la nondiscrimination, c'est-à-dire la réduction des inégalités socio-économiques entre minorités et majorité ${ }^{45}$. Ils distinguent ainsi une conception «libérale», appuyée sur une approche formaliste, où la discrimination est appréhendée comme une action intentionnelle et individualisée d'un discriminateur vers une discriminé, d'une conception plus sociologique et groupale (group-centered approach) où la discrimination n'est plus conçue comme un acte individuel ponctuel intentionnel, mais à travers ses «conséquences » (les inégalités socio-

${ }^{41}$ Éric Agrikoliansky, « Usages choisis du droit : le service juridique de la ligue des droits de l'homme (19701990) », Sociétés contemporaines, 52, 2003, p. 61-84.

${ }^{42}$ E. Fillion, D. Torny, « De la réparation individuelle à l'élaboration d'une cause collective », art. cité.

${ }^{43}$ Max Weber, Sociologie du droit, Paris, PUF, 2007.

${ }^{44}$ Nicholas Pedriana, Robin Stryker, « The Strength of a Weak Agency : Enforcement of Title VII of the 1964 Civil Rights Act and the Expansion of State Capacity, 1965-1971 », American Journal of Sociology, 110 (3), 2004, p. 709-760 ; Nicholas Pedriana, Robin Stryker, « From Legal Doctrine to Social Transformation ? Comparing US Voting Rights, Equal Employment Opportunity, and Fair Housing Legislation », American Journal of Sociology, 123 (1), 2017, p. 86-135.

${ }^{45}$ Cf. aussi Daniel Sabbagh, L'égalité par le droit. Les paradoxes de la discrimination positive aux États-Unis, Paris, Economica, 2003. 
Chappe Vincent-Arnaud, Keyhani Narguesse, « La fabrique d'un collectif judiciaire. La mobilisation des cheminots marocains contre les discriminations à la SNCF ", Revue française de science politique, 2018/1 (Vol. 68), p. 7-29. Version pré-print

économiques objectivables) et comme un processus collectif et systémique ayant des effet sur un groupe minoritaire ${ }^{46}$. Le droit étasunien, à l'origine d'inspiration libérale, serait devenu plus collectif, grâce à l'action conjuguée des mouvements sociaux, de l'Equal Employment Opportunity Commission et de savoirs des sciences psychologiques et sociales internalisés dans le droit et les entreprises ${ }^{47}$. Cette approche n'aurait que peu pénétré en France ${ }^{48}$, malgré l'introduction récente de la notion de discrimination indirecte, qui facilite théoriquement le passage à l'approche collective, dans la mesure où elle permet la prise en compte de pratiques ou mesures « en apparence neutre » mais qui ont des avantages disproportionnés sur un groupe protégé ${ }^{49}$.

L'opposition argumentative entre les avocats des plaignants et de la SNCF renvoie à cette opposition : du côté des plaignants, l'avocate met surtout en avant l'ensemble des conséquences liées au statut (approche par les conséquences), plus que le caractère volontairement discriminatoire du statut PS25. Ce n'est pas une intégration au statut de cadre permanent qui est demandée, mais un aplanissement des différences liées à ce statut. L'avocate recourt à une argumentation mixte pour soutenir ces revendications d'égalité : elle s'appuie sur des arguments moraux évoquant la reconnaissance et la dignité mais aussi le mérite, sur des normes juridiques «fondamentales » ou en tout cas à haute valeur principielles (constitution, droit et conventions internationales), et sur la « promesse » juridique de l'égalité de traitement inscrite dans le contrat de travail initial signé par les salariés. En deuxième instance particulièrement, l'avocate inscrit sa plaidoirie dans la filiation de l'histoire récente des contentieux de nondiscrimination visant à une interprétation extensive de la notion de discrimination, notamment en matière de discrimination syndicale et sexiste ${ }^{50}: \mathrm{C}$. de Lesquen a auparavant rencontré dans un événement public une consœur (Emmanuelle Boussard-Verrechia) et un syndicaliste (François Clerc) tous deux spécialisés sur ce contentieux, s'appuie sur la notion de discrimination indirecte et sur de la jurisprudence en relation. Cette filiation est également marquée par la présence d'un avocat à l'audience, Slim Ben Achour, représentant du défenseur des droits pour l'occasion, et qui lui-même détient une expérience importante dans cette matière juridique.

De l'autre côté, l'argument principal de l'avocat de la SNCF est que le traitement des Marocains est conforme au droit. Il met en avant le statut particulier de la SNCF, sorte d'îlot

46 Laure Bereni, Vincent-Arnaud Chappe, «La discrimination, de la qualification juridique à l'outil sociologique », Politix, 94, 2011, p. 7-34.

${ }^{47}$ Frank Dobbin, Inventing Equal Opportunity, Princeton, Princeton University Press, 2009 ; Robin Stryker, «L'intermédiation scientifique dans la mise en œuvre des lois anti-discrimination américaines », Droit et régulations des activités économiques. Perspectives sociologiques et institutionnalistes, Paris, LGDJ, 2011, p. 183-202.

${ }^{48}$ Frank Dobbin, «Do the Social Sciences Shape Anti-Discrimination Practice ? The United States and France », Comparative Labor Law and Policy Journal, 23 (3), 2002, p. 829-863.

${ }^{49}$ Marie Mercat-Bruns, Emmanuelle Boussard-Verrecchia, « Appartenance syndicale, sexe, âge et inégalités : vers une reconnaissance de la discrimination systémique ? », Revue de droit du travail, 11, 2015, p. 660-671.

${ }^{50}$ Vincent-Arnaud Chappe, « Dénoncer en justice les discriminations syndicales : contribution à une sociologie des appuis conventionnels de l'action judiciaire », Sociologie du travail, 55 (3), 2013, p. 302-321. 
Chappe Vincent-Arnaud, Keyhani Narguesse, « La fabrique d'un collectif judiciaire. La mobilisation des cheminots marocains contre les discriminations à la SNCF », Revue française de science politique, 2018/1 (Vol. 68), p. 7-29. Version pré-print

juridique dont le règlement intérieur est produit par le Parlement ${ }^{51}$. L'entreprise aurait parfaitement respecté le formalisme et la procédure, dont les conséquences - bien que reconnues à demi-mot comme dommageables - sont légales :

"L'avocat de la SNCF admet que "personne ne conteste que les Marocains ont bien travaillé", mais c'est une "problématique juridique et pas du tout de qualité de travail". Le débat est donc à nouveau placé sur la question du droit, de la légalité dans sa définition formelle, cherchant donc à mettre à distance le questionnement sur la qualité du travail. » (Journal de terrain du 24 mars 2015)

L'idée soutenue par les avocats de la SNCF est donc finalement que l'entreprise ferroviaire est tenue par le droit : l'argument s'inscrit ainsi dans une conception typiquement formaliste du droit de la non-discrimination où la non-intention de discriminer compte plus que la réalité effective d'une différenciation entre groupes.

La décision du juge départiteur en première instance montre la possibilité pour la justice française de s'aligner sur une conception à la fois collective et matérielle de la discrimination, même si elle pose question sur d'autres points. La rédaction du jugement pose le qualificatif de discrimination indirecte. De plus, il reconnaît la discrimination pour la quasi-totalité des plaignants, n'excluant qu'une partie des cheminots pour des raisons techniques de prescription : le tribunal se refuse ainsi à entrer dans une logique de cas par cas (a contrario de ce qu'on observe parfois dans les procès collectifs en discrimination syndicale). En même temps, la question de l'indemnisation souligne les limites de ce jugement, et finalement ce qui peut être interprété comme une forme de malaise de la justice devant ce type de procès collectif. En effet, l'indemnisation est globalement faible, et individualisée a minima : elle est calculée de façon très basique en fonction du nombre d'années d'ancienneté au sein de la SNCF - multiplié par le nombre d'années de discrimination - mais sans autre critère de distinction (par exemple concernant l'évolution effective de la carrière, le fait d'avoir passé des formations, etc.). Les dommages et intérêts prennent la forme d'un « forfait annuel » de $5000 €$ par an, multipliés par le nombre d'années de discrimination avec des sommes oscillant entre 150000 et $230000 €$, c'est-à-dire à peu près la moitié des sommes initiales demandées. Le calcul du manque à gagner est très éloigné des standards en matière de procès discrimination le long de carrière, qui procèdent habituellement par une comparaison diachronique des évolutions de salaire entre le plaignant et un groupe témoin ${ }^{52}$. Cette façon de faire montre la difficulté qu'a l'institution judiciaire à gérer les tensions entre une lecture collective de l'événement et une individualisation des réparations selon les spécificités individuelles des victimes. On constate également le caractère étonnant de l'absence de dommages et intérêts pour préjudice moral, qui atteste d'une forme de disqualification institutionnelle de la violence de la discrimination, ramenée ici à une question de manque à gagner. Pour le procès en appel, l'avocate a procédé une individualisation plus poussée des demandes, calcul qui a été pris en compte par la Cour,

\footnotetext{
${ }^{51}$ Il faut noter que, malgré l'interpellation répétée de certains députés classés à gauche de l'échiquier politique, les gouvernements successifs se sont toujours refusés à agir dans cette affaire, prétextant de l'indépendance de la compagnie ferroviaire.

52 Vincent-Arnaud Chappe, «La preuve par la comparaison: méthode des panels et droit de la nondiscrimination », Sociologies pratiques, 23, 2011, p. 45-55.
} 
Chappe Vincent-Arnaud, Keyhani Narguesse, « La fabrique d'un collectif judiciaire. La mobilisation des cheminots marocains contre les discriminations à la SNCF », Revue française de science politique, 2018/1 (Vol. 68), p. 7-29. Version pré-print

celle-ci ayant également reconnu cette fois-ci le préjudice moral ${ }^{53}$.

Le procès participe bien ainsi à la production d'une forme de collectif, réuni autour d'un même dispositif et pour lequel est produit une narration partagée en matière de discrimination. Le fonctionnement judiciaire révèle néanmoins une tension dans le traitement de cette affaire, dont le caractère collectif n'est reconnu qu'implicitement. Il faut maintenant analyser la façon dont ce groupe aboutit effectivement à un collectif unifié - et parlé - autour d'un « nous », selon quelles logiques et avec quelles contraintes.

\section{Le collectif judiciaire au risque de la subjectivation}

Au-delà des effets propres du procès sur la traduction de l'expérience des discriminations, quels sont les effets de l'ensemble du processus judiciaire sur le groupe des plaignants ? Peut-on parler d'effets d'empowerment sur le long terme par l'acquisition de nouvelles compétences politiques et d'une conscience plus aiguë des droits grâce à la mobilisation ${ }^{54}$ ? Dans sa phase associative et syndicale, la mobilisation a formé et unifié un groupe de plaignants. Mais si les cheminots marocains ont pu devenir un groupe de plaignants, c'est en devenant un groupe « raconté » par d'autres. Les différentes audiences renforcent ce processus : spectateurs, ils assistent au récit de leur propre histoire, traduite dans le langage du droit par leur avocate, et remise en cause par les avocats de la SNCF. Loin d'avoir favorisé des logiques d'investissement dans le collectif et la mobilisation, c'est plutôt à un renforcement de la frontière entre professionnels du droit et profanes ${ }^{55}$ que semble avoir contribué l'action judiciaire.

Pourtant, cette monopolisation de l'action par les professionnels du droit n'est ni absolue, ni définitive. L'organisation de l'action peut être remise en question par des plaignants jusquelà restés en retrait. Cette évolution oblige à adopter une lecture circonstanciée des effets du droit en termes de conscientisation ${ }^{56}$. Lors du procès en appel, les plaignants ont pu intervenir directement, ces prises de parole contribuant à une subjectivation des plaignants (A). En marge des audiences, l'action se poursuit et son entretien est d'autant plus nécessaire qu'elle peut être remise en cause dans son organisation par les plaignants. L'entretien de la division du travail qui caractérise l'action opère alors comme un puissant frein à sa politisation (B). Enfin, l'ethnographie au long cours de l'action judiciaire montre que lorsque les acteurs du collectif sortent du rôle qui leur a été assigné, le groupe s'expose à des risques d'éclatement qui peuvent remettre en cause son existence même $(\mathrm{C})$.

\footnotetext{
${ }^{53}$ Marie Peyronnet, «Affaire Chibanis : évaluation du préjudice résultant de la discrimination », Dalloz actualité, 31 janvier 2018, https://www.dalloz-actualite.fr/flash/affaire-chibanis-evaluation-du-prejudice-resultant-dediscrimination\#.Wovx9qjOWM8

${ }^{54}$ M. W. McCann, Rights at Work..., op. cit.

55 Éric Agrikoliansky, «Les usages protestataires du droit», dans Éric Agrikoliansky, Isabelle Sommier, Olivier Fillieule (dir.). Penser les mouvements sociaux, Paris, La Découverte, 2010, p. 225-243.

56 Stuart A. Scheingold, The Politics of Rights. Lawyers, Public Policy, and Political Change, New Haven, Yale University Press, 1974 ; M. W. McCann, Rights at Work..., op. cit..
} 
Chappe Vincent-Arnaud, Keyhani Narguesse, « La fabrique d'un collectif judiciaire. La mobilisation des cheminots marocains contre les discriminations à la SNCF », Revue française de science politique, 2018/1 (Vol. 68), p. 7-29. Version pré-print

Les effets propres d'un procès hors norme : victimisation et subjectivation

Le processus judiciaire dans son ensemble est ponctué par des moments permettant aux plaignants de prendre la mesure de l'ampleur du groupe qu'ils forment. En mars 2015, des plaignants avaient déjà pu prendre la parole pour exprimer leurs attentes quant à l'action judiciaire que l'on peut résumer comme celle d'une "assignation publique assumée de la responsabilité $\gg{ }^{57}$. Mais il s'agissait de prises de parole à l'extérieur du tribunal de prud'hommes, devant les caméras et micros de chaînes de télévision et de radio. Les quatre audiences en appel de mai 2017 ont été l'occasion pour quatre plaignants de s'exprimer directement. L'espace particulier des salles d'audience autorise à y voir un effet de légitimation collective des plaignants. C'est la Cour d'appel qui organise ces prises parole en son sein même et contrairement à ses habitudes, comme le rappelle la juge à chaque audience, présentant cette opportunité comme une faveur. C'est donc là même où leur avocate et ceux de la SNCF se sont longuement exprimés, que quatre plaignants prennent la parole pour quelques minutes seulement et deviennent de fait les porte-parole ponctuels de plaignants jusque-là spectateurs. Ces prises de parole permettent de cerner comment le groupe se définit lui-même ${ }^{58}$ pour la première fois, et ce dans un espace où est en jeu son statut de victime. Elles montrent que s'atteler à convaincre de la pertinence d'une telle qualification pour soi ne condamne pas à la passivité dans laquelle les «victimes » sont d'ordinaires campées ${ }^{59}$.

Les témoins ont été choisis en fonction de leur capacité avérée et connue des membres du bureau d'Ismaïlia ou de C. de Lesquen à prendre la parole en public. Parmi les intervenants, on compte un membre du bureau de l'association, militant CGT, mobilisé depuis le début des années 2000, ayant participé aux négociations pour la réécriture du statut en 2004 ; un autre a également une longue expérience associative et syndicale, il a déjà eu l'occasion de présenter la mobilisation devant des journalistes. Loin d'être spontanées, ces prises de parole s'inscrivent dans la stratégie de l'avocate de rendre visible individuellement les plaignants, de faire entendre leur voix par la juge, tout en les encadrant. Chaque témoin articule à des degrés divers histoire individuelle et expérience collective de la discrimination et oppose la dignité des plaignants à l'indignité de leur employeur mis en cause. L'un d'entre eux évoque une responsabilité assignée aux cheminots marocains par leurs familles pour représenter une nation entière et souligne l'écart avec le rôle qui leur est assigné à la SNCF, en tant que travailleurs immigrés :

«Le premier témoin raconte qu'au Maroc, tout le monde lui demande d'être un "bon travailleur" car il représente le Maroc en France. Pourtant, une fois arrivé, il est sommé de "travailler comme un mouton". Comme ils étaient célibataires, ils étaient considérés comme à disposition de la SNCF. Ils n'ont pas le droit de passer des examens. Mais ils ne se plaignent pas car ils ont des familles à nourrir et ont très peur des licenciements. Ils baissent donc toujours la tête. » (Journal de terrain, audience d'appel du 15 mai 2017)

S'il est plus offensif, le second témoignage s'inscrit dans la même veine et décrit des cheminots marocains porteurs d'une éthique de travail, fiers de leur enrôlement à la SNCF. Mais il exprime également sa colère à l'encontre des avocats de la défense, et rappelle

\footnotetext{
${ }^{57}$ E. Fillion, D. Torny, « De la réparation individuelle à l'élaboration d'une cause collective », art. cité, p. 592.

${ }^{58}$ R. Brubaker, «Au-delà de l'“identité" », art. cité.

${ }^{59} \mathrm{~S}$. Lefranc, L. Mathieu, Mobilisations de victime, op. cit.
} 
Chappe Vincent-Arnaud, Keyhani Narguesse, « La fabrique d'un collectif judiciaire. La mobilisation des cheminots marocains contre les discriminations à la SNCF ", Revue française de science politique, 2018/1 (Vol. 68), p. 7-29. Version pré-print

l'importance pour les plaignants de voir leur employeur reconnu comme responsable par une institution judiciaire qui par sa solennité impressionne le porte-parole temporaire :

«Le second témoin s'adresse à "Mme la Présidente, mesdames messieurs les jurés". Il veut parler de son vécu car la SNCF veut échapper à ses responsabilités. Elle est pourtant venue les chercher au Maroc. Il se dit très vexé par l'idée qu'ils ne parlaient pas français et rappelle qu'ils ont passé des tests de langue au Maroc. Il met au défi la SNCF de dire qu'elle les avait prévenus qu'ils seraient traités différemment [...]. Elle les a parqués et a profité d'eux. Lui a écrit au ministère, qui l'a renvoyé vers les RH qui prétend ne pas avoir de pouvoir [...]. Ils étaient pourtant très fiers de travailler à la SNCF, il se souvient de discussions avec des collègues : « les rails de France sont les meilleurs du monde !». Car leur travail était rigoureux, un travail qu'ils ont appris et transmis ». Sa voix finit par trembler, il retient ses larmes. Il dit lui-même être très ému et trouve dommage que ce qui est dit à l'oral ne soit pas pris en compte, mais il tenait à témoigner. » (Journal de terrain, audience du 15 mai 2017)

Cette seconde intervention illustre bien comment la prise de parole pour les plaignants est l'occasion de faire entendre une histoire collective au-delà du parcours personnel. Elle décrit aussi les raisons qui font d'un PS25 un plaignant : avant de le devenir, le porte-parole a frappé à toutes les portes pour demander justice.

Le caractère réactif de la contestation se donne également à voir dans le cadre temporel des audiences : les interventions des deux dernières audiences peuvent apparaissent comme la mobilisation de ce droit à la parole publique pour réagir à ce qui dans les plaidoiries de la défense a été perçu comme une offense. Le troisième témoin lit son intervention. Notre interlocuteur privilégié nous expliquera que malgré le manque de spontanéité, il s'agissait de répondre à l'argument utilisé la veille par la défense mettant en avant l'illettrisme supposé des PS25. La dernière intervention, plus théâtrale, est par ailleurs plus organisée :

«Le dernier témoin commence par se dire gêné et honoré de se retrouver devant "Madame la présidente", c'est "inédit pour [lui]" et il remercie tout le monde, notamment le défenseur des droits. Son exposé est organisé en 4 points. "Premier point : la discrimination étant institutionnalisée à la SNCF"; deuxième point : "l'avocat de la SNCF a sous-entendu que les auxiliaires avaient beaucoup d'enfants". Il demande alors "si les contrats signés dans les années 1970 exigeaient des cheminots stériles ?" (la salle est hilare). [...]. Troisièmement, si le passage d'auxiliaire à cadre permanent était possible pour ceux devenus français, les évolutions n'étaient pas comparables. Enfin, la SNCF dit ne pas être responsable du calcul des pensions, mais elle l'était en amont, à travers les salaires versés. Il conclut : "quand on dit "cadre permanent", ça signifie "permanent dans la prospérité et les avantages". Les auxiliaires permanents eux étaient permanents dans la lenteur de l'avancement, permanent dans la médiocrité. » (Journal de terrain, audience du 16 mai 2017)

Si elle provoque l'hilarité de la salle, cette dernière intervention montre l'importance pour le plaignant devenu porte-parole l'espace d'un instant, de donner à voir sa maîtrise du langage mais aussi des arguments de la SNCF qu'il juge offensants pour le portrait dépréciatif qu'il y lit en filigrane. C'est un imaginaire collectif faisant du travailleur immigré venu des anciennes colonies le représentant d'un groupe illettré et aux caractéristiques démographiques incontrôlables qu'il remet en cause par son trait d'humour et sa connaissance des règles de gestion du personnel. Ces interventions ne sont pas la répétition identique des plaidoiries : elles peuvent être lues comme une réaction à la «victimisation secondaire » comprise comme une réaction à la non-reconnaissance de leur statut de victime ${ }^{60}$, en l'occurrence par les avocats de la défense. Prendre la parole au cœur même de l'institution judiciaire, même ponctuellement permet d'intervenir comme acteur du processus judiciaire qui, s'il contribue à la victimisation

\footnotetext{
${ }^{60}$ Y. Barthe, Les retombées du passé..., op. cit., p. 107.
} 
Chappe Vincent-Arnaud, Keyhani Narguesse, « La fabrique d'un collectif judiciaire. La mobilisation des cheminots marocains contre les discriminations à la SNCF », Revue française de science politique, 2018/1 (Vol. 68), p. 7-29. Version pré-print

souhaitée des plaignants, a jusque-là été portée par d'autres acteurs ${ }^{61}$. Ces moments restent rares mais en raison de l'espace particulier dans lequel ils surviennent et des applaudissements chaleureux qu'ils suscitent, ils participent à valoriser les plaignants devenus «victimes accusatrices $\gg 62$.

Mais cet équilibre autour des porte-parole temporaires reste précaire. Des divisions font surface au sein du collectif qui renvoient à la définition même du porte-parolat. À la fin des audiences, dans la cour du tribunal, des membres du bureau et le dernier intervenant discutent et évaluent les différentes interventions. Certains reprochent l'émotivité de l'un d'entre eux, alors que d'autres apprécient l'expression publique de ces émotions et estiment qu'elle aura peut-être marqué la juge. Ce qui est en jeu, c'est la maîtrise de l'oral comme ressource nécessaire pour représenter le groupe en public.

\section{La division du travail comme frein à la politisation}

Au-delà de l'espace spécifique du procès, l'action judiciaire peut être plus largement porteuse de dynamiques amenant à redéfinir le rôle des acteurs dans la mobilisation. Par son organisation, celle des cheminots marocains semble freiner une éventuelle dynamique de désingularisation et de publicisation ${ }^{63}$.

On peut l'observer à un premier niveau, celui de l'organisation de l'action et de la répartition des rôles. La stricte division du travail entre association, professionnels du droit et plaignants répond certes à une logique de rationalisation de la gestion parallèle de centaines de plaintes. Mais elle présente aussi d'autres aspects. Tout d'abord les professionnels du droit ont adopté une stratégie de dépolitisation assumée, qui se donne à voir par la mise à distance d'autres acteurs. L'association Droits à la différence crée par Radouane Kebdi, un ancien cheminot beaucoup plus jeune du cadre permanent tente depuis quelques années en s'appuyant sur l'affaire des cheminots marocains, d'articuler dénonciation des discriminations subies par les travailleurs immigrés arrivés en France pendant les Trente Glorieuses et celles subies par les descendants d'immigrés au sein de l'entreprise. Cette montée en généralité pour dénoncer les discriminations touchant plusieurs générations passe notamment par une importante publicisation de l'affaire sur les réseaux sociaux et des médias en ligne, mais aussi par la proximité avec certains plaignants. Tenu informé des différentes audiences et des Assemblée générale, R. Kebdi est présent et suit l'affaire de près. La réaction des organisateurs est simple et radicale : elle refuse tout simplement sa présence physique. Il est poussé vers la sortie lors des assemblées et rabroué publiquement pendant le procès des prud'hommes :

«Nous assistons à une altercation entre Radouane et quelques cheminots, qui semble faire suite à une autre altercation dans le hall. On lui reproche d'avoir organisé un rassemblement de soutien à l'extérieur du tribunal. Un homme notamment, à côté de A. Katim, lui demande de quel droit il utilise le mot Chibani, et le menace même d'appeler la police. La dispute nous semble sévère. » (Journal de terrain, audience du 23 mars 2015)

${ }^{61}$ M. Avanza, G. Laferté, « Dépasser la “construction des identités” ?.. », art. cité.

${ }^{62}$ Jean-Noël Jouzel, Giovanni Prete, «De l'intoxication à l'indignation : le long parcours d'une victime des pesticides », Terrains \& travaux, 22, 2013, p. 59-76.

${ }^{63}$ Camille Hamidi, «Éléments pour une approche interactionniste de la politisation », Revue française de science politique, 56 (1), février 2006, p. 5-25. 
Chappe Vincent-Arnaud, Keyhani Narguesse, « La fabrique d'un collectif judiciaire. La mobilisation des cheminots marocains contre les discriminations à la SNCF », Revue française de science politique, 2018/1 (Vol. 68), p. 7-29. Version pré-print

Cette volonté, ferme, d'entretenir un entre soi, compris comme face-à-face exclusif avec les plaignants et de contrôler strictement les règles d'entrée aux assemblées générale permet de cerner la définition extérieure du groupe selon les organisateurs : il est destiné à rester un groupe de plaignants et non à devenir un collectif où plaignants et soutiens échangeraient, et pourraient potentiellement diversifier les modes d'action par l'entremise de soutiens extérieurs ${ }^{64}$ ou faire émerger d'autres conflits. Cela éclaire les fonctions que ces organisateurs semblent donner à ces assemblées générales. Organisées à la Bourse du travail, elles sont l'une des rares occasions de rencontre pour l'ensemble des protagonistes ${ }^{65}$ : les membres du bureau d'Ismaïlia, A. Bendali et l'avocate peuvent revenir sur l'action et répondre aux questions de l'ensemble des plaignants. Contrairement aux audiences, l'interaction réunit les plaignants et leurs soutiens et les premiers peuvent prendre la parole. À la différence des permanences, C. de Lesquen est présente et peut rencontrer l'ensemble des plaignants, même si de fait un quart seulement y participe (environ 200 personnes). Mais comme pour les permanences, la disposition de la salle sépare les organisateurs (côté tribune), des plaignants (côté public).

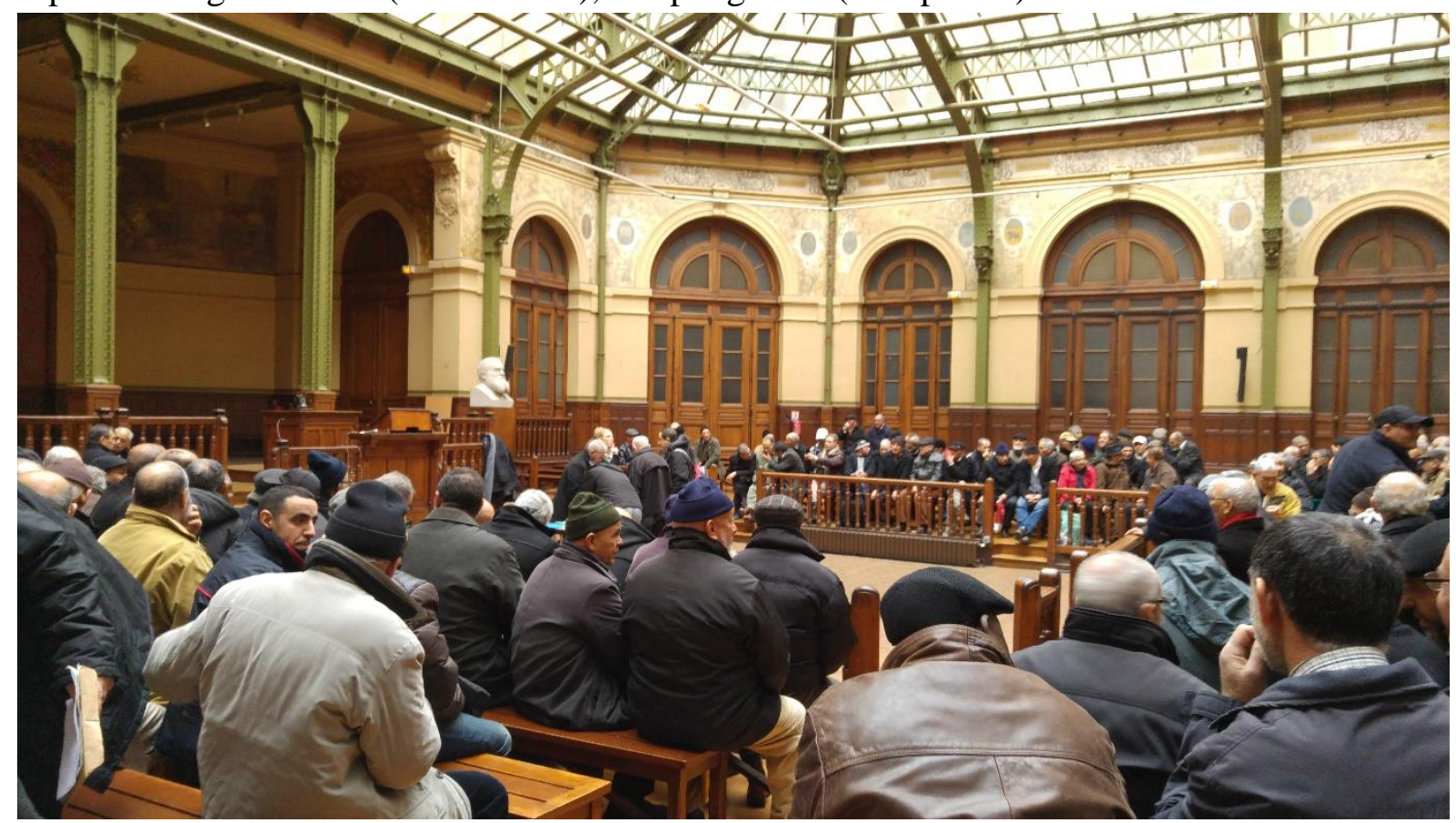

Bien que rares, leur fonctionnement est relativement routinisé. C'est l'occasion pour les plaignants d'entendre celui que bon nombre considèrent comme le chef d'orchestre de l'action judiciaire : alternant le français et l'arabe ${ }^{66}$, « le professeur » revient sur le travail qu'il a mené avec l'avocate, soulignant le temps consacré à chaque dossier, le « sacrifice » consenti en laissant de côté leurs autres dossiers et leur temps libre. « 1600 pages », « $4 \mathrm{~m}^{3}$ de dossiers ! » :

\footnotetext{
${ }^{64}$ Choukri Hmed, «Contester une institution dans le cas d'une mobilisation improbable : la "grève des loyers" dans les foyers Sonacotra dans les années 1970 », Sociétés contemporaines, 65, 2007, p. 55-81.

${ }^{65}$ Observations des 18 janvier et 11 avril 2016, et du 23 mai et 11 décembre 2017.

${ }^{66} \mathrm{~N}$ 'étant pas arabophones, nous avons demandé à notre interlocuteur privilégié de traduire ses interventions, qui restent néanmoins parcellaires.
} 
Chappe Vincent-Arnaud, Keyhani Narguesse, « La fabrique d'un collectif judiciaire. La mobilisation des cheminots marocains contre les discriminations à la SNCF ", Revue française de science politique, 2018/1 (Vol. 68), p. 7-29. Version pré-print

le travail est quantifié et rappelé pour s'assurer la reconnaissance des plaignants. C'est aussi l'occasion de remémorer les règles établies au fil de l'action judiciaire : la stricte division des tâches et la nécessité de passer par lui ou l'association pour toute question plutôt que de chercher à contacter directement l'avocate. Mais à côté des rappels à l'ordre, il s'agit aussi de rassurer les plaignants, désarçonnés par la longueur de l'action judiciaire. L'avocate intervient pour expliquer cette longueur et les rassurer en mettant en scène sa compétence :

"Accueillie par de chaleureux applaudissements, l'avocate rassure l'assemblée en rappelant qu'après la condamnation aux prud'hommes: "c'est aux appelants d'expliquer pourquoi ils ne sont pas d'accord". Mais pendant ce temps "on va étoffer notre dossier". Elle précise qu'elle plaidera après les appelants : "C'est toujours mieux de parler en dernier". Elle les rassure quant à l'horizon de cette longue action : "on aura bientôt une date, fin 2016, début 2017". Elle insiste enfin sur l'importance de rester unis et d'être massivement présents : "Chaque personne a un calcul qui lui est propre, mais chacun bénéficie du travail collectif de l'association [...]. C'est important que vous soyez là, nombreux. Je l'ai vu dans le regard du juge, ça l'a marqué que vous soyez tous là, que vous soyez venus de toute la France, certains du Maroc.” » (Journal de terrain, 18 janvier 2016)

En traduisant en langage courant les règles de fonctionnement de l'institution judiciaire, elle s'en fait la traductrice et entretient la fiction d'un combat collectif auquel tout le monde participerait à parts égales. Ces interventions qui visent à justifier l'organisation de l'action en en rappelant le bien-fondé répondent aussi à un souci de régulation des tensions dont témoigne l'évocation systématique des prises de contact avec l'avocate, présentées comme des tentatives de court-circuiter l'association.

Remise en cause de l'organisation et fragilisation du collectif

Les assemblées font apparaître des tensions au sein du groupe de plaignants. Les activités de l'association semblent parfois insuffisamment ordonnées et explicitées aux yeux des plaignants. Lors des assemblées, certains commentent sa faible communication qui de façon récurrente les prévient tardivement de la tenue des rencontres en oubliant certains d'entre eux ; d'autres soulignent qu'une association est censées rédiger annuellement des rapports d'activités et les diffuser à ses cotisants, que des assemblées doivent être organisées de façon beaucoup plus régulières et qu'il faut mieux encadrer les tours de parole. Surtout la question des sommes d'argent demandées par l'association est récurrente : entre eux ou en posant directement la question aux organisateurs, cette question cristallise d'importantes tensions.

Elle catalyse un nombre croissant d'interrogations qui au cours de l'année 2017 ont pris une ampleur particulièrement forte. De simples questions sur la nature des frais, la demande de clarification s'est muée en remise en cause de l'organisation. Pour le comprendre, il faut d'abord rappeler le rapport ambivalent des plaignants à cette question. Si par définition elle tient une place centrale dans la mobilisation, l'action judiciaire visant concrètement une réparation financière, tous les entretiens avec les plaignants de même que leurs interventions publiques font apparaître une tension entre d'une part le souhait évident de voir l'injustice « réparée » financièrement, et d'autre part la mise à distance de cet objectif : les plaignants disent souhaiter la reconnaissance publique de la discrimination et nient la primauté de leur intérêt financier. Les travaux qui interrogent les effets politisant des actions judiciaires, tendent à faire de la distanciation avec les enjeux financiers un critère central de la possibilité de montée 
Chappe Vincent-Arnaud, Keyhani Narguesse, « La fabrique d'un collectif judiciaire. La mobilisation des cheminots marocains contre les discriminations à la SNCF », Revue française de science politique, 2018/1 (Vol. 68), p. 7-29. Version pré-print

en généralité d'une action ${ }^{67}$. Ici, le rapport à l'argent, dans les coulisses de l'action judiciaire, renvoie à la position relative des plaignants et de leurs soutiens dans la mobilisation. Ainsi, après les audiences en appel de mai 2017, un clivage semble avoir vu le jour au sein du groupe autour de plusieurs enjeux : la rémunération de l'avocate, celle de A. Bendali, les sommes demandées par l'association à l'occasion de la préparation des dossiers. Les deux premiers sont intimement liés : la question du pourcentage à verser à l'avocate pour chaque dossier a fait surface lorsqu'a été posée celle de la rémunération de A. Bendali. Le fait que ce dernier, n'étant pas avocat n'ait aucun statut légal dans la conduite de l'action a compromis la possibilité de le rémunérer, rémunération acquise pour de nombreux plaignants du fait de sa centralité à leurs yeux dans l'action judiciaire. Un conflit est alors né entre des plaignants, tous souhaitant une clarification des rôles des uns et des autres. Celui de l'association s'en est retrouvé interrogé face au manque de clarté perçu par des plaignants quant aux sommes demandées. Ces tensions ont alors pris la forme de tensions de plus en plus explicites, des plaignants souhaitant recourir à un autre avocat, d'autres souhaitant poursuivre avec le conseil ayant fait condamner la SNCF, d'autres enfin s'interrogeant sur le statut réel et souhaitable de A. Bendali. En l'état actuel, il est difficile d'objectiver plus précisément les termes du conflit et les différents griefs, mais il autorise d'ores et déjà quelques pistes d'interprétation. Une première renvoie à la faible bureaucratisation de l'association (absence de traces écrites systématiques, importance de l'oralité par rapport à l'écrit dans l'établissement des règles d'organisation de l'action). Une seconde renvoie aux plaignants : dotés dans l'ensemble de faibles capitaux scolaires limitant de fait leur compétences procédurales ${ }^{68}$, ils ont remis leur action à d'autres, sans véritablement maîtriser les tenants et aboutissants de l'organisation de l'action judiciaire, méconnaissant par ailleurs les règles de fonctionnement du champ des professionnels du droit (celles déterminant la rémunération d'un avocat, celles relatives à la rémunération d'un tiers sans statut dans l'action judiciaire).

Ce que fait apparaitre ce conflit, c'est la dépendance de la trajectoire de l'action judiciaire aux premières étapes de sa construction. Ce sont les conditions mêmes de l'organisation du groupe des plaignants comme groupe unifié qui permettent de comprendre la crise dont des linéaments semblent aujourd'hui se dessiner : la fragilité du groupe qui se donne à voir avec le risque de son éclatement, renvoie à celle de l'association, pourtant moteur de l'action collective et judiciaire. La prise de parole, la volonté de s'approprier l'action en en saisissant mieux l'organisation, la revendication d'avoir son mot à dire dans la définition des relations entre les différentes parties, autrement dit les facteurs qui dans d'autres contextes peuvent signaler des dynamiques de participation, semblent ici mettre en danger le collectif. Lors d'une assemblée où ces clivages se sont exprimés avec force, un plaignant s'inquiète :

«On ne parle plus de notre affaire : il faut qu'on en parle !» ${ }^{69}$

67 J.-P. Vilain, C. Lemieux, « La mobilisation des victimes d'accidents collectifs... », art. cité ; .M. W. McCann, Rights at Work..., op. cit., chap. 7.

${ }^{68}$ Alexis Spire et Katia Weidenfeld, «Le tribunal administratif: une affaire d'initiés ? Les inégalités d'accès à la justice et la distribution du capital procédural », Droit et Société, vol. 79 / 3, 2011, p. 689-713.

${ }^{69}$ Notes de terrain, 11 décembre 2017. 
Chappe Vincent-Arnaud, Keyhani Narguesse, « La fabrique d'un collectif judiciaire. La mobilisation des cheminots marocains contre les discriminations à la SNCF ", Revue française de science politique, 2018/1 (Vol. 68), p. 7-29. Version pré-print

La mobilisation judiciaire a bien des effets de conscientisation chez ces plaignants, conscientisation qui passe notamment par l'identification entretenue à un groupe de salariés discriminés en raison de leur origine et de leur nationalité. L'inscription de l'action judiciaire dans la durée, parce qu'elle nécessite l'actualisation des dossiers individuels et qu'elle favorise l'accroissement du groupe des plaignants, alimente par ailleurs cette conscientisation en renforçant la socialisation des plaignants entre eux et en favorisant la production de récits semblables sur ce qu'a été l'expérience de la discrimination et sur ce devrait être sa réparation (avant tout être reconnue et par la justice et par la SNCF). Cette approche collective de la discrimination a également pu être portée dans l'arène plus strictement judiciaire bien que l'institution en rende la défense difficile. Mais à travers la mobilisation judiciaire et le procès, ce sont bien des discriminations collectives qui ont été qualifiées.

L'ethnographie du procès et de la mobilisation judiciaire plus généralement mettent pourtant également en évidence une dépossession de fait du mouvement par les formes mêmes de l'action judiciaire : l'organisation des permanences et la violence symbolique exercée sur les plaignants par les professionnels du droit en sont le signe le plus manifeste. La conscientisation par l'action judiciaire ne s'accompagne pas ici strictement d'un processus de grandissement. Les explications se trouvent du côté des caractéristiques socio-démographiques des plaignants, faiblement dotés en capitaux et ressources, et des motivations des entrepreneurs de mobilisation, peu soucieux de faire du procès un levier d'empowerment individuel ou collectif.

Pour autant, les plaignants se manifestent dans l'arène judiciaire comme en témoignent les prises de parole spontanées devant les médias après les audiences ou au cours des réunions publiques pour demander plus de transparence sur le travail de l'association et plus d'information sur le cours du procès. Si ces prises de paroles ne suffisent pas à politiser la mobilisation, elles attestent de la volonté de maîtriser ne serait-ce que l'information.

Enfin, le procès et sa médiatisation suscitent de nouvelles mobilisations, comme par exemple le lancement récent d'une pétition en ligne et d'une interpellation directe du président de la SNCF Guillaume Pépy par des enfants de cheminots, l'utilisation de ce verdict par l'association La Fédération des maisons des potes pour tenter de rouvrir le dossier de l'accessibilité des emplois publics aux étrangers extra-communautaires, ou le lien effectué par l'association Droit à la différence avec le Front Uni des immigrations et des quartiers populaires (FUIQP) pour monter en généralité sur la question des discriminations subies par les populations immigrées des anciennes colonies et leurs descendants. Ces tentatives de penser ensemble et joindre des mobilisations différenciées témoignent des «effets de radiation» (radiating effects ${ }^{70}$ ) des mobilisations judiciaires, susceptibles de faire émerger de nouveaux

\footnotetext{
${ }^{70}$ Marc Galanter, « The Radiating Effects of Courts », dans Keith D. Boyum, Lynn Mather, Empirical Theories of Courts, New York, Longman, 1983, p. 117-142.
} 
Chappe Vincent-Arnaud, Keyhani Narguesse, « La fabrique d'un collectif judiciaire. La mobilisation des cheminots marocains contre les discriminations à la SNCF ", Revue française de science politique, 2018/1 (Vol. 68), p. 7-29. Version pré-print

collectifs et sujets d'action dans l'espace public ${ }^{71}$.

\section{Vincent-Arnaud Chappe et Narguesse Keyhani}

Sociologue, chargé de recherche au CNRS (section 36), Vincent-Arnaud Chappe est membre du Centre de sociologie de l'innovation (CSI-I3, Mines ParisTech, PSL Research University). Il a récemment participé (avec Mireille Eberhard et Cécile Guillaume) à la coordination d'un numéro de Terrains \& Travaux portant sur «La fabrique des discriminations » $(29,2016)$, et publié (avec Cécile Guillaume et Sophie Pochic) dans Travail et Emploi un article intitulé «Négocier sur les carrières syndicales pour lutter contre la discrimination : une appropriation sélective et minimaliste du droit» $(145,2016$, p. 121-146). Il travaille sur les politiques d'égalité et de lutte contre les discriminations au travail, à partir de deux directions : les contentieux judiciaires en matière de discrimination dans leur dimension collective ; les appuis statistiques dans le cadre des politiques d'égalité femmes-hommes au sein des entreprises (CSII3, Mines ParisTech, 60 boulevard Saint-Michel, 75006 Paris, <vincentarnaud.chappe @ mines-paristech.fr >).

Docteure en sociologie, affiliée à l'Institut des sciences sociales du politique (ISSP) et actuellement post-doctorante au Centre d'études de l'emploi et du travail, Narguesse Keyhani a notamment publié « Former pour dépolitiser : l'administration des immigrés comme cible de l'action publique », Gouvernement et action publique, 4 (4), 2012, p. 91-114; et « La "question des races" dans un cadre administratif républicain : la création de la Commission nationale pour les études des relations interethniques », Cultures \& Conflits, 107 (3), 2017, p. 61-76. Ses recherches actuelles portent sur les mobilisations collectives et l'action publique contre le racisme et les discriminations (CEET, 29 Promenade Michel Simon, 93160 Noisy-le-Grand, <narguesse_keyhani@yahoo.fr>).

La fabrique d'un collectif judiciaire. La mobilisation des cheminots marocains contre les discriminations à la SNCF

L'article porte sur la mobilisation des cheminots marocains, engagés dans les années 1970 par la SNCF comme contractuels, et ayant fait condamner l'entreprise ferroviaire pour discrimination. L'ethnographie du processus judiciaire explore les relations entre mobilisations et action judiciaire et leurs effets sur la formation des collectifs. En revenant sur les conditions d'une action au long cours, exceptionnelle par le nombre de plaignants, et sur les modalités de sa traduction judiciaire, il montre que la judiciarisation participe à la constitution de ce collectif, mais qu'elle fait apparaître une tension entre conscientisation des plaignants et dépossession par les professionnels du droit.

Mots clés : mobilisation judiciaire, collectif de plaignants, victimes, discrimination au travail,

71 Nous remercions Marion Charpenel, Édouard Gardella, Julien Talpin, l'équipe « droit, mobilisation, discrimination » coordonnée par Liora Israël dans le cadre du financement «Émergence » de la Mairie de Paris, pour les différents commentaires et conseils apportés sur des versions intermédiaires de cet article. Nous remercions également tous nos enquêtés pour leur aide précieuse, et plus particulièrement Bob Frahlia. Les propos tenus n'engagent néanmoins que leurs auteurs. 
Chappe Vincent-Arnaud, Keyhani Narguesse, « La fabrique d'un collectif judiciaire. La mobilisation des cheminots marocains contre les discriminations à la SNCF ", Revue française de science politique, 2018/1 (Vol. 68), p. 7-29. Version pré-print

procès, immigration.

The making of a judicial collective. The mobilization of Moroccan railway workers against discrimination at SNCF

The article deals with the mobilization of Moroccan railway workers. The French national train company hired them as contract workers in the 1970s. They achieved to sentence the company for discrimination. The ethnography of the judicial process explores the relationships between mobilization and legal action and their effects on the formation of a collective. The conditions of a long-term action (which was exceptional because of the number of complainants) and of its judicial translation, show that judicialization participated in the constitution of this group. This process reveals a tension between conscientization of complainants and dispossession by legal professionals.

Keywords: Judicial Mobilization, Plaintiffs' Collective, Victims, Discrimination at Work, Litigation, Immigration. 